\title{
THE NONLINEAR EFFECTS OF FRINGE FIELDS IN HLS*
}

\author{
Lin Wang, Zuping Liu, Guangyao Feng, Shancai Zhang, Weimin Li, Hongliang Xu \\ NSRL of USTC, P.R.China
}

\begin{abstract}
The main body of HLS (Hefei Light Source) is an $800 \mathrm{MeV}$ electron storage ring, whose circumference is about $66 \mathrm{~m}$. As a typical small ring, although the sextupole fields for correction of chromaticities are relatively weak, there still exist strong nonlinear fields coming from fringe fields of dipole and quadrupole magnets. In this paper, the effects of fringe fields on chromaticities and tune shifts with amplitude were discussed and calculated for HLS storage ring. Under the low injection energy, the tune shifts with amplitude coming from fringe fields are one of important sources of transverse Landau damping, which is main stabilizing mechanism for HLS ring.
\end{abstract}

\section{INTRODUCTION}

HLS (Hefei Light Source) is a dedicated synchrotron radiation research facility, spectrally strongest in VUV and soft X-ray. The operation energy of storage ring is $800 \mathrm{MeV}$, while injection energy is $200 \mathrm{MeV}$ only. There are total 12 dipoles in the ring, whose deflecting angle is 30 degree. The transverse radiation damping time under operation energy is about $20 \mathrm{~ms}$, while under injection energy it is very slow, approximately $1.4 \mathrm{~s}$. As a second generation light source, the vacuum chamber were not specially designed for optimization of beam coupling impedance. At HLS, there haven't other reliable beam stabilizing measures, such as longitudinal and transverse feedback systems, non-uniform filling mode, except for Landau damping, which is coming from transverse tune spread and becomes a main stabilizing mechanism at storage ring. Usually, the second order effects of sextupoles, which used to compensate chromaticities or to enlarge dynamic aperture, would result in tune shift with amplitude in transverse planes ${ }^{[2,3]}$ :

$$
\left\{\begin{array}{l}
v_{x}=v_{x 0}+a \cdot J_{x}+b \cdot J_{y} \\
v_{y}=v_{y 0}+b \cdot J_{x}+c \cdot J_{y}
\end{array}\right.
$$

For HLS storage ring, there exists another source of tune shift with amplitude - fringe fields of main magnets. Different methods were used in description and analysis of fringe field effects, for example, [4] describes the fringe field effects in small rings of large acceptance; [5], [6] and [7] give the description and analysis of quadrupole fringe field effects by hard edge model; [8] gives the transfer map of dipole, quadrupole and solenoid fringe fields; [9] derived the description of the soft fringe field of quadrupole. In this paper, the effects of dipole and quadrupole fringe fields on chromaticities and tune shift with amplitude were analyzed and calculated by Lie

\footnotetext{
*Work supported by National Natural Science Foundation of China (10575096)

\#wanglin@ustc.edu.cn
}

algebra method.

\section{HAMILTONIAN WITH FRINGE FIELDS}

For large storage ring, the fringe field effects are neglected generally and the Hamiltonian for main magnet can be expressed as :

$$
\begin{aligned}
& H\left(x, p_{x}, y, p_{y}, \delta, l ; s\right) \approx-\delta+\frac{\left(p_{x}-a_{x}\right)^{2}}{2(1+\delta)} \\
& +\frac{\left(p_{y}-a_{y}\right)^{2}}{2(1+\delta)}-a_{z}
\end{aligned}
$$

Assuming piecewise-constant model of the magnetic field of dipole, quadrupole, sextupole and octupole magnets, the vector potentials $\boldsymbol{a}_{\boldsymbol{x}}$ and $\boldsymbol{a}_{\boldsymbol{y}}$ can be set as zero. So, vector potential for main magnets is

$$
\begin{aligned}
& a_{z}=-\frac{1}{2}\left(1+\kappa_{x} \cdot x\right)^{2}+\frac{1}{2} K \cdot\left(y^{2}-x^{2}\right)-\frac{1}{6} \lambda . \\
& \left(x^{3}-3 x y^{2}\right)-\frac{1}{24} \mu \cdot\left(y^{4}-6 x^{2} y^{2}+x^{4}\right)
\end{aligned}
$$

It is easy to know that above vector potential met the requirement of Maxwell equations. Actually, in the fringe field region of magnets, the magnetic field and vector potential are dependent on the longitudinal position $\boldsymbol{S}$. So, according to Maxwell equation, the vector potential can't be expressed by (3) simply. In the fringe field region, magnetic field and vector potential have higher order components, which are dependent on the longitudinal distribution of magnetic field $\boldsymbol{B}_{y}(\boldsymbol{z})$. Some papers derived mathematical expression of magnetic field and vector potential in fringe field region, in cylindrical coordinates ${ }^{[10]}$ or in Frenet-Serret coordinates ${ }^{[11]}$. For HLS storage ring, the deflecting angle of dipole is large and the R K Symon's expressions for vector potential (4), which was truncated at fourth order, were employed in this paper. The truncation of this formula would not affect the purpose of this paper, to study the effects of fringe field on chromaticities and tune shift with amplitude. From vector potential of quadrupole and dipole, the effects from fringe fields can be estimated, that the fringe field of quadrupole would result in tune shift with amplitude, and the fringe field of dipole are likely to produce chromaticities and tune shift with amplitude.

$$
\left\{\begin{array}{l}
a_{x}=-\frac{K^{\prime}(s)}{2} x y \\
a_{y}=0 \\
a_{s}=\frac{K(s)}{2}\left(y^{2}-x^{2}\right)+\frac{K^{\prime \prime}(s)\left(x^{4}-6 x^{2} y^{2}+y^{4}\right)}{48}
\end{array}\right.
$$




$$
\begin{aligned}
& \int a_{x}=-\frac{B_{1} \cos (\alpha)}{2} y^{2}+\frac{B_{2} \sin (\alpha) \cos (\alpha)}{2} x y^{2} \\
& \begin{array}{l}
-\frac{B_{3} \sin ^{2}(\alpha) \cos (\alpha)}{4} x^{2} y^{2}+\frac{B_{3} \cos (\alpha)}{24} y^{4} \\
a_{y}=0
\end{array} \\
& \left\{\begin{array}{l}
a_{s}=-B x+\left(\frac{B_{1} \sin (\alpha)}{2}-\frac{B}{2 \rho}\right) x^{2}- \\
\frac{B_{1} \sin (\alpha)}{2} y^{2}+\left(\frac{B_{1} \sin (\alpha)}{3 \rho}-\frac{B_{2} \sin ^{2}(\alpha)}{6}\right) x^{3}
\end{array}\right. \\
& +\left(\frac{B_{2} \sin ^{2}(\alpha)}{2}-\frac{B_{1} \sin (\alpha)}{2 \rho}\right) x y^{2} \\
& +\left(\frac{B_{3} \sin ^{3}(\alpha)}{24}-\frac{B_{2} \sin ^{2}(\alpha)}{8 \rho}\right) x^{4} \\
& +\left(\frac{B_{2} \sin ^{2}(\alpha)}{2 \rho}-\frac{B_{3} \sin ^{3}(\alpha)}{4}\right) x^{2} y^{2}+\frac{B_{3} \sin (\alpha)}{24} y^{4} \\
& \int_{1}=\frac{B^{\prime}(s)}{\cos (\alpha)} \\
& B_{2}=\frac{B^{\prime \prime}(s)}{\cos ^{2}(\alpha)}-\frac{B^{\prime}(s) \sin (\alpha)}{\rho \cos ^{3}(\alpha)} \\
& B_{3}=\frac{B^{\prime \prime \prime}(s)}{\cos ^{3}(\alpha)}-\frac{3 B^{\prime \prime}(s) \sin (\alpha)}{\rho \cos ^{4}(\alpha)}+ \\
& \frac{B^{\prime}(s)\left(1+2 \sin ^{2}(\alpha)\right)}{\rho^{2} \cos ^{5}(\alpha)}-\frac{B^{\prime 2}(s) \sin (\alpha)}{(B \rho) \cos ^{4}(\alpha)} \\
& \rho(s)=\frac{(B \rho)}{B(s)} \quad \frac{d \alpha(s)}{d s}=-\frac{1}{\rho(s)}
\end{aligned}
$$

\section{DESCRIPTION OF FRINGE FIELDS}

In the piecewise-constant approximation, the higher order differentials of $\boldsymbol{K}(s)$ or $\rho(s)$, which used to describe the higher order components, are zero and (4) was reduced to (3). In some codes, step function was used to characterize the longitudinal distribution of magnetic field and its higher order differentials are not zero. For simplicity, approximate transfer map of fringe field was used in particle tracking. In reality, the longitudinal distribution of magnetic field is dependent on the geometry of magnet. Finishing the physical design of magnets or the measurement of magnetic field, the detail of longitudinal distribution is known. More accurate analysis and calculation of fringe field effects are possible. Firstly, a mathematical function to describe longitudinal distribution of magnetic field is needed. Due to orthogonality, any function can be expressed as orthogonal series, whose coefficients can be obtained by numerical fitting ${ }^{[12]}$. For simplicity, the Enge function ${ }^{[4,}$ ${ }^{13]}$ was used to describe distribution of magnetic field.

$$
G(s)=\frac{1}{1+\operatorname{Exp}\left(a_{0}+a_{1} \frac{s}{d}+a_{2}\left(\frac{s}{d}\right)^{2}+a_{3}\left(\frac{s}{d}\right)^{3}+\cdots\right)}
$$

The sym $\boldsymbol{d}$ is the diameter of magnets and the sym $\boldsymbol{a}_{\boldsymbol{i}}$ are coefficients obtained by numerical fitting. The Enge functions with 6 coefficients were used to fit the longitudinal distribution of magnetic field in the fringe region of quadrupole and dipole. The fitting process was made by MATLAB and the fitting errors are very small. Figure 1 and figure 2 displayed the longitudinal distribution of field in dipole and quadrupole.

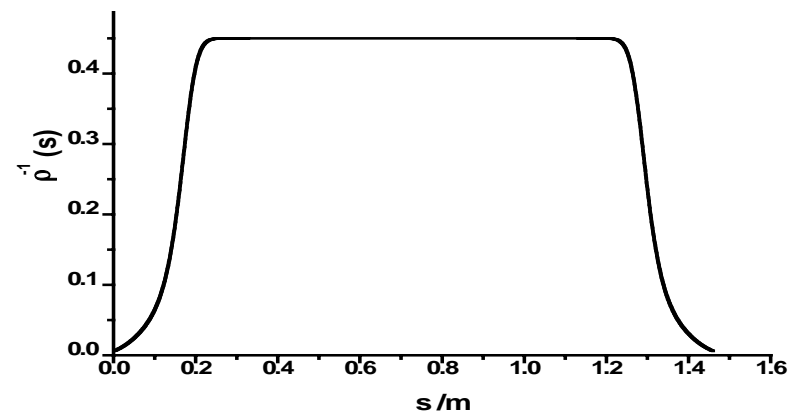

Figure 1: Longitudinal distribution of curvature in dipole

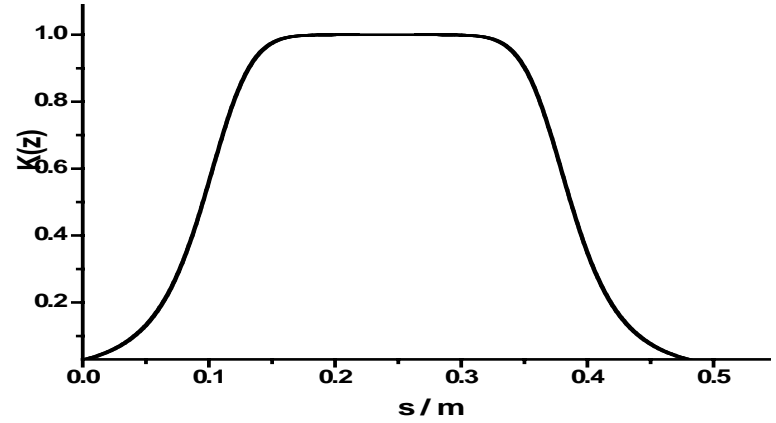

Figure 2: Longitudinal distribution of $\boldsymbol{K}(s)$ in quad

According to fitted Enge function, higher order differentials of $K(s)$ and $\rho(s)$ can be calculated and the detailed vector potential are known. The table 1 gives the fitted coefficients of Enge functions.

Table 1: Fitted Values of Enge Function

\begin{tabular}{|l|l|l|l|l|l|l|}
\hline & $\boldsymbol{a}_{\mathbf{0}}$ & $\boldsymbol{a}_{\mathbf{1}}$ & $\boldsymbol{a}_{\mathbf{2}}$ & $\boldsymbol{a}_{\mathbf{3}}$ & $\boldsymbol{a}_{\mathbf{4}}$ & $\boldsymbol{a}_{\mathbf{5}}$ \\
\hline Quad & 0.2334 & -4.798 & -2.231 & -1.167 & -0.1198 & -0.0194 \\
\hline Dipole & 0.3997 & 2.054 & -0.714 & -0.193 & -0.0041 & 0.0 \\
\hline
\end{tabular}

\section{LIE ALGEBRA TOOLS}

Knowing the Hamiltonians of magnets with fringe fields, there are many tools to analyze its effects theoretically or to track particle trajectory numerically. The Lie algebra provide powerful tools to obtain the chromaticity, tune shifts with amplitude, momentum compaction factor and nonlinear driving terms, or to extract numerical symplectic or non-symplectic transfer map used in tracking. Due to the dependence of Hamiltonian on $\boldsymbol{s}$, some tricks are needed to perform analysis and tracking, for example, extending to the generalized 8-d canonical space ${ }^{[2]}$. Here, a simple method was adopted. The fringe field region was divided to $n$ slices, where the Hamiltonian is assumed independent of coordinate $s$ and its value is dependent on the slice 
number only. A magnet with fringe field is replaced by a series of small magnetic element, which contained various order components in (4). Then, the $\mathrm{BCH}$ and concatenation formulae were used to extract effective Hamiltonian of magnet with fringe field or whole ring, which can be used to deduce the Taylor transfer map directly by truncation or to obtain the chromaticities, tune shift with amplitude, momentum compaction factor and resonance driving terms by normal form analysis. After normal form transformation, the second order part of effective Hamiltonian includes transverse tunes and linear momentum compaction factor, and the third order part can be expressed as ${ }^{[14]}$

$$
h_{3}=C_{0000,3} \delta^{3}+C_{1100,1} J_{x} \delta+C_{0011,1} J_{y} \delta,
$$

Where the first term is second order momentum compaction factor, the second and third terms are linear horizontal and vertical chromaticities. The four order part is as following

$$
\begin{aligned}
& h_{4}=C_{0000,4} \delta^{4}+C_{1100,2} J_{x} \delta^{2}+C_{0011,2} J_{y} \delta^{2} \\
& +C_{2200,0} J_{x}^{2}+C_{1111,0} J_{x} J_{y}+C_{0022,0} J_{y}^{2}
\end{aligned}
$$

And it contains the third order momentum compaction, second order chromaticities and linear tune shift with amplitude. From (7) it is easy to obtain (1).

\section{FRINGE FIELD EFFECTS FOR HLS}

From normal form transformation process, it is found that fringe field of quadrupole is similar to octupole components and contribute to tune shift with amplitude, and the fringe field of dipole contains sextupole-like and octupole like components and contributes to linear chromaticities and tune shift with amplitude. Also, calculation showed that there exists small difference of transverse tunes between soft magnet model and hard edge model, which is on account of limited extension of fringe field. The table 2 gives the main calculation results. The detailed numerical transfer map and tracking results can refer to [15].

Table 2: Main Calculation Results

\begin{tabular}{|l|l|l|l|}
\hline & \multicolumn{2}{|l|}{ Natural Chromaticity } \\
\hline Without fringe of dipole & \multicolumn{2}{|l|}{$-6.2 /-4.3$} \\
\hline With fringe of dipole & \multicolumn{2}{|l|}{$-6.2 /-6.3$} \\
\hline & \multicolumn{2}{|l|}{ Tune shift with amplitude } \\
\cline { 2 - 4 } & $\boldsymbol{a}$ & $\boldsymbol{b}$ & $\boldsymbol{c}$ \\
\hline With 4-th order kinetic terms & 6.1 & 3.6 & 4.8 \\
\hline Adding fringe of quad & 137.8 & 44.75 & 64.9 \\
\hline Adding fringe of dipole & 138.7 & 48.75 & 273.7 \\
\hline Adding sextupoles & 169.3 & -161.7 & 257.2 \\
\hline
\end{tabular}

\section{CONCLUSION}

It is not suppressing that, fringe fields of dipole and quadrupole have significant contributions to linear chromaticities and tune shift with amplitude, and became one of main sources of Landau damping.

\section{REFERENCES}

[1] National Synchrotron Radiation Laboratory of University of Science and Technology of China, "Development report of Hefei synchrotron radiation accelerator "(in Chinese), 1991.

[2] E.Forst, "Beam Dynamics: A New Attitude and Framework", Harwood academic publishers, 1998.

[3] L.Tosi, V.Smaluk, E.Karantzoulis, "Landau damping via the harmonic sextupole”, PRST-AB Vol. 6, 054401(2003).

[4] M.Berz, B.Erdelyi, K.Makino, "Fringe field effects in small rings of large acceptance”, PRST-AB Vol. 3, 124001(2000).

[5] F.Zimmermann, "Tune shift with amplitude induced by quadrupole fringe fields”, CERN-SL-2000009(AP).

[6] E.Forest, "Leading order hard edge fringe fields effects exact in $(1+\delta)$ and consistent with Maxwell's equations for rectilinear magnets”, SSC-142, 1987.

[7] M.Venturini, "Scaling of third-order quadrupole aberrations with fringe field extension”, Proceedings of PAC99, p1590-1592.

[8] Y.Cai, Y.Nosochkov, "Dynamical effects due to fringe field of the magnet in circular accelerators", SLAC-PUB-11181, 2005.

[9] J.Irwin, C.Wang, "Explicit soft fringe maps of a quadrupole”, Proceedings of PAC95, p2376-2378.

[10] R.M.G.M. Trines, S.J.L. van Eijndhoven, J.I.M.Botman, et al, "Modeling the fields of magneto-optical devices, including fringe field effects and higher order multipole contributions, with application to charged particle optics", PRSTAB Vol.4, 062401(2001).

[11] K.R.Symon, "Derivation of Hamiltonians for accelerators”, Argonne National Laboratory, 2005.

[12] K.Balewski, W.Decking, Y.Li, "Beam dynamics study for PETRA III including damping wigglers", Proceedings of EPAC04, p1999-2001.

[13] H.A.Enge, "Effect of extended fringing fields on ion-focusing properties of deflecting magnets", THE REVIEW OF SCIENTIFIC INSTRUMENTS, 1964.

[14] A.W.Chao, "Lecture notes on topics in accelerator phyisics”, SLAC-PUB-9574, 2002.

[15] L.Wang, "The beam dynamics study of Hefei Light Source storage ring”(in Chinese), PhD, Univ. S.\& T. China, 2006. 